\title{
The Relative Proportion of Preterm Births Complicated by Premature Rupture of Membranes in Multifetal Gestations: A Population-Based Study
}

\author{
Tarita Pakrashi, MD, MPH ${ }^{1}$ Emily A. Defranco, DO, MS ${ }^{1}$ \\ ${ }^{1}$ Department of Obstetrics and Gynecology, University of Cincinnati, \\ Cincinnati, Ohio, \\ Address for correspondence and reprint requests Tarita Pakrashi, \\ MD, MPH, Department of Obstetrics and Gynecology, University of \\ Cincinnati College of Medicine, Medical Sciences Building, 231 Albert \\ Sabin Way, Cincinnati, OH 45267-0526 \\ Am J Perinatol 2013;30:69-74. \\ (e-mail: pakrast@ucmail.uc.edu).
}

\begin{abstract}
Keywords

- PROM

- preterm/premature birth

- multifetal gestation

- preterm rupture of membranes

- preterm labor

Objective To compare the relative contribution of premature rupture of membranes (PROM) to preterm births in singleton compared with multifetal gestations.

Study Design A population-based retrospective cohort study of 291,782 nonanomalous live births in the state of Ohio from January 1, 2006, to December 31, 2007, identified through birth certificate data. Frequency of PROM was compared between singleton and multifetal gestations and then stratified by gestational age at birth. Multivariate regression analysis estimated the risk of PROM by plurality in each gestational age group, accounting for influential risk factors.

Results The frequency of PROM increased with increasing plurality of gestation. The relative proportion of preterm birth $<37$ weeks complicated by PROM increased with gestational plurality $13.2 \%$ singletons, $16.8 \%$ twins, $20.0 \%$ triplets, $19.6 \%$ quadruplets, and $100 \%$ for higher-order multiples $(p<0.001)$. The frequency of PROM increased with earlier gestational age at birth, regardless of plurality. The increased risk of PROM in multifetal gestation persisted even after adjustment for influential concomitant risk factors.

Conclusion The proportion of preterm birth attributable to PROM increases by plurality of gestation, with its most significant contribution in higher-order multiples and at earliest gestational ages when outcomes are the poorest.
\end{abstract}

Preterm birth is a profound public health issue. Despite a decrease in the mortality rate after extremely preterm birth, the morbidity is increasing. ${ }^{1}$ Preterm births contribute to $75 \%$ of all perinatal mortality and more than half the long-term morbidity in infant survivors. ${ }^{2}$ It has been estimated that $\sim 30$ to $35 \%$ of preterm births are medically indicated, 40 to $45 \%$ are attributed to spontaneous preterm labor, and 25 to $30 \%$ follow preterm premature rupture of membranes (PROM). ${ }^{3}$

Based on U.S. birth records, Martin et al found that in 2006, one of every eight twins and one of three triplets were born at less than 32 weeks compared with 1 of 50 singletons. ${ }^{4}$ The rates of preterm birth at $<37$ weeks of gestation and at $<32$ weeks of gestation are $11.1 \%$ and $1.6 \%$ for singletons, $92.6 \%$ and $12.1 \%$ for twins, $92.6 \%$ and $36.3 \%$ for triplets, $94.9 \%$ and $79.2 \%$ for quadruplets, and $89.6 \%$ and $79.1 \%$ for quintuplet pregnancies, respectively. ${ }^{4}$

PROM is defined as rupture of the amniotic membranes before the onset of labor. Preterm PROM occurs at less than 37 weeks of gestation. PROM occurs in 1 to $2 \%$ of pregnancies nationally and is reported to recur in $21 \%$ of cases. ${ }^{5}$ It is well known that PROM is associated with an increase in complications of pregnancy such as cord prolapse, placental received

December 30, 2011

accepted after revision

March 15, 2012

published online

July 6, 2012
Copyright $\odot 2013$ by Thieme Medical Publishers, Inc., 333 Seventh Avenue, New York, NY 10001, USA. Tel: +1(212) 584-4662.
DOI http://dx.doi.org/ 10.1055/s-0032-1321502. ISSN 0735-1631. 
abruption, chorioamnionitis, and preterm birth. A Swedish study examining the role of preterm delivery and neonatal outcome in a population of infants born at $<28$ weeks of gestation reported that infant survival was significantly lower when preterm delivery was secondary to PROM versus spontaneous preterm labor with intact membranes or indicated delivery. ${ }^{6}$ Another study examining PROM between 17 to 30 weeks of gestation demonstrated a significant increase in pulmonary hypoplasia and symptomatic chronic lung disease when compared with a control group of infants born secondary to spontaneous preterm labor. ${ }^{7}$ These studies highlight the importance of recognizing the risk of PROM especially at early gestational ages when preterm neonates are the most vulnerable to complications of prematurity.

Some studies have noted that multifetal gestation is a risk factor for PROM, and it is hypothesized that uterine overdistension is the probable cause of PROM in these pregnancies. ${ }^{8,9}$ Although some studies have described an increase in the incidence of PROM in twin gestations in comparison with singleton pregnancies, there are no current large populationbased epidemiological studies that have examined the relative proportion of preterm births attributed to PROM, stratified by plurality of gestation.

\section{Methods}

The protocol for this study was approved by the Ohio Department of Health and Human Subjects Institutional Review Board, and a deidentified data set was provided by the Child Policy Research Center of Cincinnati Children's Hospital Medical Center. This study was exempt from review by the Institutional Review Board at the University of Cincinnati, Cincinnati, Ohio.

We performed a population-based retrospective cohort study using the Ohio Department of Health's birth certificate database to study the relative contribution of PROM to preterm birth in multifetal gestations (twins, triplets, quadruplets, and higher-order multiples) compared with singleton births. PROM was defined as premature rupture of membranes $>12$ hours, as coded in the birth certificate. The relative frequency of PROM was stratified for each group with respect to gestational age at birth (term, 34 to 37 weeks, 28 to 34 weeks, and $<28$ weeks). When comparing by plurality, singletons were the referent category, and for gestational age comparisons, births occurring at term (37 to 42 weeks) were used as the reference group for comparison.

Births with gestational age $<20$ weeks, pregnancies complicated by major congenital anomalies, and fetal deaths were excluded from this study. After exclusions, there were 291,782 births remaining for this analysis. The primary outcome measure was PROM with the plurality of gestation as the exposure. The secondary outcomes studied were preterm birth, frequency and proportion of preterm births complicated by PROM, stratified by gestational age. For each of the variables included in this study, there was minimal missing data. There were no missing data on gestational age at birth. There was a single birth in the data set coded as being born to a gestation with plurality $>6$. This birth was dropped from the data set prior to analysis due to the likelihood of coding error, after taking into consideration characteristics of the birth that excluded the possibility it was indeed a higherorder multifetal gestation. There were only 16 births with missing data on plurality of the gestation $(<0.01 \%$ missing $)$ and 4873 births with missing information on PROM (1.7\%). Likewise, there were minimal missing data on demographic characteristics studied ( $\leq 3 \%$ for all variables except no prenatal care with $11 \%$ missing data).

Statistical analysis was performed using STATA (Stata Statistical Software: Release 10. College Station, TX: StataCorp LP). Demographic characteristics between the groups were compared using the analysis of variance test for continuous variables and chi-square test for categorical variables. Comparisons with $p$ value $<0.05$ or $95 \%$ confidence interval (CI) not inclusive of the null were considered statistically significant. Multivariate logistic regression estimated the risk of PROM by gestational age at birth and plurality. Covariates were chosen for the final regression model by differences noted in bivariate analysis, significance testing through backward elimination, and parsimony within the model.

\section{Results}

There were 291,782 live births included in this analysis. Of those, 280,882 (96.3\%) were singletons, 10,293 (3.5\%) twins, $539(0.2 \%)$ triplets, $46(0.02 \%)$ quadruplets, and $6(0.01 \%)$ higher-order multiples. The frequency of PROM by gestational plurality is illustrated in - Table 1. PROM occurred more frequently in twins (11.2\%), triplets (19.3\%), quadruplets

Table 1 Distribution of PROM by Gestational Plurality $(n=291,782)$

\begin{tabular}{|l|l|l|}
\hline & PROM $^{\mathrm{a}}(\boldsymbol{n}=\mathbf{9 8 7 6} ; \mathbf{3 . 4 \% )}$ & No PROM $(\boldsymbol{n}=\mathbf{2 7 7 , 0 1 7 ;} \mathbf{9 6 . 6 \%}$ \\
\hline Singletons, $n(\%)$ & $8607(3.1)$ & $267,584(96.9)$ \\
\hline Twins, $n(\%)$ & $1150(11.2)$ & $8,970(88.8)$ \\
\hline Triplets, $n(\%)$ & $104(19.3)$ & $426(79.7)$ \\
\hline Quadruplets, $n(\%)$ & $9(19.6)$ & $37(79.4)$ \\
\hline Higher order $(>4), n(\%)$ & $6(100)$ & $0(0)$ \\
\hline
\end{tabular}

PROM, premature rupture of membranes.

${ }^{\mathrm{a}}$ The difference in rate of PROM, stratified by plurality of the gestation, was statistically significant, $p<0.001$.

There were $4873(1.7 \%)$ births with missing data on PROM and $16(<0.01 \%)$ with missing data on plurality, resulting in $n=286,893$ for this comparison. 
Table 2 Maternal Characteristics of Pregnancies Complicated by PROM, by Gestational Plurality $(n=9876)$

\begin{tabular}{|l|l|l|l|l|l|l|}
\hline & Singleton & Twin & Triplet & Quadruplet & Higher Order & $p$ Value \\
\hline Parity & $1.8 \pm 1.3$ & $2.3 \pm 1.3$ & $2.2 \pm 1.1$ & $3.2 \pm 1.4$ & $3.5 \pm 1.9$ & $<0.001^{\mathrm{a}}$ \\
\hline Age of mother (y) & $26.7 \pm 6.2$ & $30.0 \pm 5.8$ & $32 \pm 5.9$ & $28 \pm 5$ & $23 \pm 0$ & $<0.001^{\mathrm{a}}$ \\
\hline AMA, age $>35$ & $1110(12.9)$ & $199(17.3)$ & $33(31.7)$ & 0 & 0 & $<0.001^{\mathrm{b}}$ \\
\hline Race & & & & & & $<0.001^{\mathrm{b}}$ \\
\hline White & $6300(73.8)$ & $902(78.9)$ & $98(94.2)$ & $9(100)$ & $6(100)$ & \\
\hline Black & $1628(19)$ & $189(16.5)$ & $6(5.8)$ & 0 & 0 & \\
\hline Hispanic & $361(4.2)$ & $30(2.6)$ & 0 & 0 & 0 & \\
\hline Other & $253(3)$ & $24(2)$ & 0 & 0 & 0 & \\
\hline Tobacco use & $1925(22.6)$ & $176(15.3)$ & $4(4)$ & $5(55.6)$ & 0 & $<0.001^{\mathrm{b}}$ \\
\hline No PNC & $299(4.1)$ & $16(1.9)$ & 0 & 0 & 0 & $0.006^{\mathrm{b}}$ \\
\hline ART & $55(1.2)$ & $77(13.3)$ & $33(47.8)$ & $4(50)$ & 0 & $<0.001^{\mathrm{b}}$ \\
\hline Prior PTB & $481(5.7)$ & $46(4.2)$ & $11(10.9)$ & 0 & 0 & $0.037^{\mathrm{b}}$ \\
\hline CHTN & $156(1.8)$ & $38(3.4)$ & $8(7.7)$ & 0 & 0 & $<0.001^{\mathrm{b}}$ \\
\hline GHTN & $350(4.2)$ & $70(6.3)$ & $6(5.8)$ & 0 & 0 & $0.016^{\mathrm{b}}$ \\
\hline Pregest DM & $114(1.32)$ & $16(1.39)$ & 0 & 0 & 0 & $0.009^{\mathrm{b}}$ \\
\hline GDM & $428(5)$ & $91(7.9)$ & $18(17.3)$ & 0 & 0 & $<0.001^{\mathrm{b}}$ \\
\hline Cerclage & $102(1.19)$ & $23(2)$ & $15(14.4)$ & 0 & 0 & $<0.001^{\mathrm{b}}$ \\
\hline Tocolysis & $409(4.8)$ & $102(8.9)$ & $19(18.3)$ & 0 & 0 & $<0.001^{\mathrm{b}}$ \\
\hline
\end{tabular}

Continuous variables are expressed as mean \pm standard deviation. Binary variables are expressed as number (percent). AMA, advanced maternal age; ART, artificial reproductive technology; CHTN, chronic hypertension; GDM, gestational diabetes mellitus; GHTN, gestational hypertension; PNC, prenatal care; Pregest DM, pregestational diabetes mellitus;PROM, premature rupture of membranes; PTB, preterm birth.

${ }^{\mathrm{a}}$ Analysis of variance $t$ test.

${ }^{\mathrm{b} C h i-s q u a r e ~ t e s t . ~}$

(19.6\%), and higher-order multiples (100\%) compared with singleton pregnancies (3.1\%; $p<0.001$ ). Multifetal gestations complicated by PROM were more likely to occur in women who were of advanced maternal age ( $>35$ years), Caucasian, recipients of artificial reproductive technology, diagnosed with chronic hypertension and gestational diabetes, and those who underwent interventions such as cerclage and tocolysis (-Table 2).

PROM occurred more frequently in multifetal gestations and at earlier gestational ages. In singleton pregnancies, the rate of PROM increased with earlier gestational age at birth. Compared with the frequency of PROM at term (1.8\%), PROM affected $10.5 \%$ of late preterm singletons (34 to 37 weeks) (adjusted odds ratio [adjOR] 5.4, 95\% CI 5.1 to 5.7 ), $18.2 \%$ born at 28 to 34 weeks (adj OR $10.8,95 \%$ CI 10 to 11.7 ), and $27.1 \%$ of extreme preterm singleton births $<28$ weeks (adj OR 18.8, 95\% CI 16.5 to 21.4). Similarly, the rate and risk of PROM increased with degree of prematurity in twins. The rate of PROM increased in twins from $2 \%$ at term to $35.7 \%$ at less than 28 weeks of gestation (adj OR 39.6, 95\% CI 27.8 to 56.5). The rate of PROM was particularly high in quadruplets and higherorder multifetal gestations born at extremely preterm gestational ages, $50 \%$ and $100 \%$, respectively (-Table $\mathbf{3}$ ).

The relative proportion of preterm births $<37$ weeks complicated by PROM increased with gestational plurality: PROM contributed to $13.2 \%$ of all preterm births $<37$ weeks in singletons, $16.8 \%$ in twins, $20.0 \%$ in triplets, $19.6 \%$ in quadruplets, and $100 \%$ in higher-order multiples $(p<0.001)$. - Figure 1 demonstrates the relative proportion of PROM in preterm births by plurality and gestational age. The relative proportion of PROM in preterm births increased with earlier gestational age at birth. At late preterm gestational ages, 34 to 37 weeks, PROM contributed to $10.2 \%$ of singleton births, $11 \%$ of twins, $12.2 \%$ of triplets, and $10 \%$ of quadruplets $(p=0.516)$. At 28 to 34 weeks, PROM contributed to $17.8 \%$ of singleton, $24.3 \%$ of twin, $23.1 \%$ triplets, and $14.3 \%$ of quadruplet births $(p<0.001)$. And at extremely preterm gestational ages, the proportion of preterm births attributable to PROM was $26.5 \%$ for singleton births, $34.6 \%$ for twins, $26.9 \%$ for triplets, $50 \%$ for quadruplets, and $100 \%$ of higher-order multifetal gestations $(p<0.001)$.

\section{Discussion}

In this study, we found that preterm birth complicated by PROM occurred with increasing frequency in multifetal gestations compared with singletons. PROM became a more frequent contributor to preterm birth in multiples as the plurality of the gestation increased. Likewise, we found that PROM occurred with increasing frequency at the earliest gestational ages in higher-order multifetal gestations, less than 28 weeks, when outcomes are most compromised. 
72 Preterm Births Complicated by PROM in Multifetal Gestations Pakrashi, Defranco

Table 3 Risk of PROM by Gestational Age, Stratified by Plurality

\begin{tabular}{|c|c|c|c|c|c|c|}
\hline & Singleton $^{\mathrm{a}}$ & Twin & Triplet & Quad & Higher Order & $p$ Value \\
\hline PROM, $n(\%)$ & 8607 & 1150 & 104 & 9 & 6 & \\
\hline $37-42 w^{a}$ & $4373(1.8 \%)$ & $73(2 \%)$ & $3(11.5 \%)$ & 0 & 0 & 0.001 \\
\hline OR & 1.0 & 1.0 & 1.0 & 1.0 & & \\
\hline $34-37$ wk & 2441 (10.5\%) & 461 (11.2\%) & $23(12.2 \%)$ & $1.0(10 \%)$ & 0 & 0.52 \\
\hline adj $\mathrm{OR}^{\mathrm{c}}$ & $5.4(5.1,5.7)$ & $6.0(4.5,8.0)$ & $1.8(0.5,6.6)$ & $\mathrm{b}$ & & \\
\hline $28-34$ wk & $1272(18.2 \%)$ & $454(24.8 \%)$ & $57(23.5 \%)$ & $4(14.3 \%)$ & 0 & $<0.001$ \\
\hline adj $\mathrm{OR}^{\mathrm{c}}$ & $10.8(10,11.7)$ & $19.1(14.2,25.6)$ & $2.2(0.6,7.9)$ & b & b & \\
\hline$<28 w k$ & $521(27.1 \%)$ & $162(35.7 \%)$ & $21(28.4 \%)$ & $4(50 \%)$ & $6(100 \%)$ & $<0.001$ \\
\hline $\operatorname{adj} \mathrm{OR}^{\mathrm{c}}$ & $18.8(16.5,21.4)$ & $39.6(27.8,56.5)$ & $7.3(1.8,29.0)$ & b & b & \\
\hline$p$ value & $<0.001$ & $<0.001$ & 0.03 & 0.08 & $\mathrm{~N} / \mathrm{A}$ & \\
\hline
\end{tabular}

Total $n$ for this population is 291,782 . N/A, not applicable; PROM, premature rupture of membranes.

a Singleton gestation and PROM at term (37-42 wk) were the reference groups used for comparison.

bUnable to calculate due to zero observations in reference group.

${ }^{\mathrm{c}}$ Covariates in the regression model include: parity, no prenatal care, race, prior preterm birth, gestational hypertension.

The complications associated with the birth of a preterm baby, especially a neonate delivered after PROM, include but are not limited to respiratory distress syndrome, intraventricular hemorrhage, neonatal sepsis, pulmonary hypoplasia, and restrictive deformation. In fact, Chen et al found that infants born following PROM had the highest risk of neonatal death compared with births attributed to the other subtypes of prematurity. ${ }^{10}$

The complex pathophysiological mechanisms leading to preterm PROM are not completely understood, but it is widely accepted that underlying infection and inflammation disproportionately affect cases of PROM, which may in part explain poorer neonatal outcomes for these births. ${ }^{11}$ Ascending uterine infection can lead to fetal infection, which may further lead to a fetal inflammatory response syndrome that is not only linked to preterm labor but also fetal morbid- ity such as periventricular leukomalacia, cerebral palsy, and chronic lung disease. $^{3}$

A population-based study similar to ours examined data from the Perinatal Information System database in Montevideo, Uruguay, where 885,338 pregnancies were studied and of which, 15,484 were multifetal gestations. ${ }^{12}$ Their study differed in that they found no increased risk of PROM in multiple gestations when compared with singleton pregnancies. ${ }^{12}$ However, the authors did not categorize multifetal gestations by order of plurality, instead classifying all pregnancies with two or more fetuses as "multiple gestation."

Ananth et al performed a retrospective cohort study of twin live births delivered in the United States by reviewing U.S. twin natality files between 1989 and 2000. They noted an increase in medically indicated preterm births in black and white populations in the United States within the

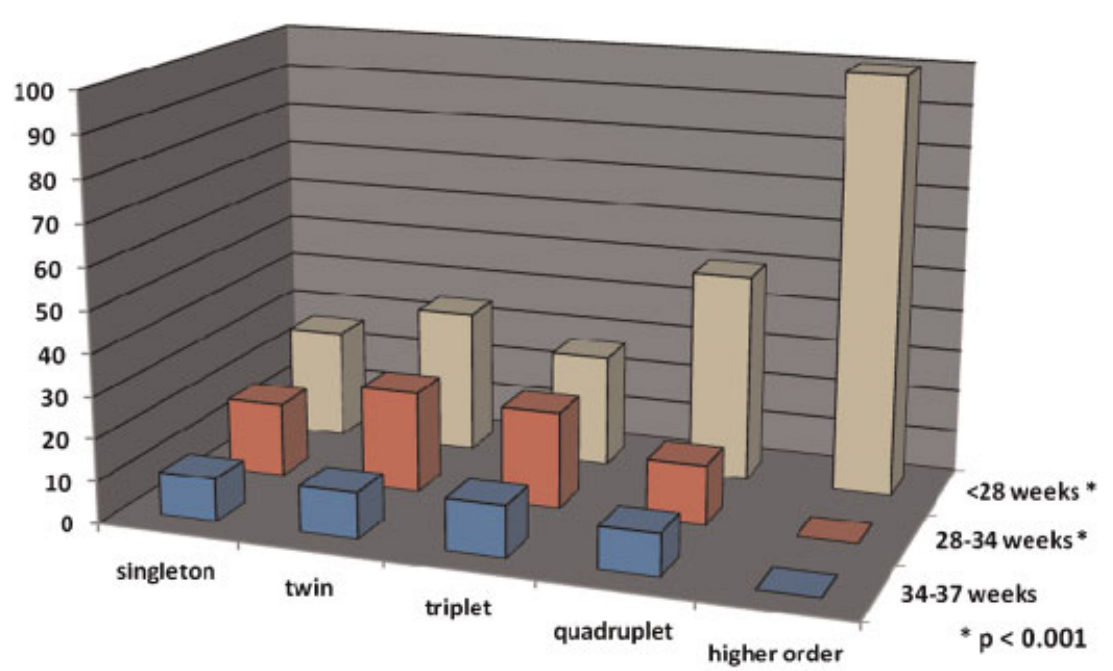

Figure 1 Relative contribution of premature rupture of membranes to preterm birth by plurality and gestational age. 
aforementioned time period. ${ }^{13}$ However, preterm births attributed to PROM remained the same in the white population and decreased in the black population in the study period. Our study differs from Ananth et al's study in that we examined the frequency of PROM in higher-order multiples greater than twins and calculated the relative risk of birth at a preterm gestational age due to PROM in each category of multifetal gestation and included all racial/ethnic groups.

This was a population-based analysis with a large sample size and hence had sufficient power to detect small differences in the relative frequency of PROM by increasing plurality of gestation, its relative proportional contribution to preterm birth, and its occurrence by gestational age. Unlike prior studies that examined a variety of maternal and perinatal outcomes in multiple births, this study focused solely on PROM and its effect on preterm birth by plurality of gestation and stratified the risks by gestational age. The other strengths of the data source used for this study include diversity and a representation of the different strata of society and physician practices that allowed for generalization.

This study demonstrated that PROM is a frequent contributor to preterm birth in singletons as well as multifetal gestations. Ours is the first study we are aware of that quantified the risk of PROM by plurality of gestation and by gestational age and its proportional contribution to preterm birth, compared with preterm birth with intact membranes. It also demonstrated that PROM affects multifetal gestations more commonly than singletons with its highest frequency occurring in higher-order multiples and at the earliest gestational ages when the outcomes are the poorest.

The limitations of this study include a low number of higher order $(>4)$ multiple gestations in the study and the inability to differentiate between spontaneous versus indicated preterm birth. In large medical databases such as this, the validity of data depends on the validity of information in the medical record and the ability of coding personnel to extract relevant patient data. ${ }^{14}$

Another drawback of analyzing information from vital statistics data is missing information. This limitation can be minimized by posing questions that do not require the use of variables with significant missing values or the use of statistical techniques to account for the absent information. ${ }^{14}$ This study avoided some of the published biases in that we chose exposure and outcomes that had minimal missing data (gestational age and plurality). We also had rates of demographic characteristics similar to reported norms and minimal missing data for variables identified. Undercoding or misclassification of specific diagnoses is common in vital statistics data. For example, a recent study from the Ohio Perinatal Quality Collaborative compared medical indications for labor induction between chart abstracted data and medical information gathered from Ohio birth certificates. ${ }^{15}$ The authors found that lack of a documented medical indication was 11 times more frequent in birth certificate data compared with data found in the patient record. This is likely due to lack of documentation of a medical indication in the birth certificate, when in fact one does exist. Similarly, we found the rate of PROM contributing to preterm birth in this study to be 10 to $15 \%$, which is considerably lower than previously published reports attributing one-third of preterm births to PROM. ${ }^{16,17}$ This likely reflects the underreporting of PROM across all gestational subtypes in the birth certificate database in Ohio. Although we do not suspect that PROM was differentially misclassified by plurality or gestational age, if it was more frequently undercoded in singletons or later gestational ages, this could result in an overestimate in our comparisons of relative proportional differences and risks among groups. Additionally, the possibility of residual confounding by unaccounted environmental and behavioral risk factors in the study also cannot be excluded with complete certainty.

It is also interesting to note that the previously mentioned and oft-quoted studies quantifying the contribution of PROM to preterm birth were performed in 1987 and 1991, respectively, and there has been a lack of recent data reflecting the contribution of PROM to preterm birth.

\section{Conclusion}

Multifetal gestations had a higher frequency of preterm birth complicated by PROM in this study. This occurred more frequently in higher-order multiples and at extremely premature gestational ages. This study provides additional information to support the important role that PROM plays in preterm birth especially in multifetal gestations and helpful evidence in counseling of this high-risk category of obstetric patients.

\section{Note}

"The relative contribution of PROM to preterm birth in multifetal compared with singleton gestations: A population-based study" was presented at the 77th Annual Meeting of the Central Association of Obstetricians and Gynecologists, October 27 to 30, 2010, Las Vegas, Nevada.

\section{References}

1 McParland P, Jones G, Taylor D. Preterm labour and prematurity. Curr Obstet Gynaecol 2004;14:309-319

2 McCormick MC. The contribution of low birth weight to infant mortality and childhood morbidity. N Engl J Med 1985;312:82-90

3 Goldenberg RL, Culhane JF, Iams JD, Romero R. Epidemiology and causes of preterm birth. Lancet 2008;371:75-84

4 Martin JA, Hamilton BE, Sutton PD, et al. Births: Final data for 2006. Natl Vital Stat Rep 2009;57:1-18

5 Hadley CB, Main DM, Gabbe SG. Risk factors for preterm premature rupture of the fetal membranes. Am J Perinatol 1990;7:374-379

6 Johanzon M, Odesjö H, Jacobsson B, Sandberg K, Wennerholm UB. Extreme preterm birth: onset of delivery and its effect on infant survival and morbidity. Obstet Gynecol 2008;111:42-50

7 Kurkinen-Räty M, Koivisto M, Jouppila P. Perinatal and neonatal outcome and late pulmonary sequelae in infants born after preterm premature rupture of membranes. Obstet Gynecol 1998;92:408-415

8 Iams JD, Stilson R, Johnson FF, Williams RA, Rice R. Symptoms that precede preterm labor and preterm premature rupture of the membranes. Am J Obstet Gynecol 1990;162:486-490 
9 Mercer BM, Crocker LG, Pierce WF, Sibai BM. Clinical characteristics and outcome of twin gestation complicated by preterm premature rupture of the membranes. Am J Obstet Gynecol 1993;168:1467-1473

10 Chen A, Feresu SA, Barsoom MJ. Heterogeneity of preterm birth subtypes in relation to neonatal death. Obstet Gynecol 2009;114: 516-522

11 Merenstein GB, Weisman LE. Premature rupture of the membranes: neonatal consequences. Semin Perinatol 1996;20; 375-380

12 Conde-Agudelo A, Belizán JM, Lindmark G. Maternal morbidity and mortality associated with multiple gestations. Obstet Gynecol 2000;95(6 Pt 1):899-904

13 Ananth CV, Joseph KS, Demissie K, Vintzileos AM. Trends in twin preterm birth subtypes in the United States, 1989 through 2000 : impact on perinatal mortality. Am J Obstet Gynecol 2005;193; (3 Pt 2):1076-1082

14 Cahill AG, Macones GA. Vital considerations for the use of vital statistics in obstetrical research. Am J Obstet Gynecol 2006;194:909-910

15 Bailit JL; Ohio Perinatal Quality Collaborative. Rates of labor induction without medical indication are overestimated when derived from birth certificate data. Am J Obstet Gynecol 2010; 203:269, e1-e3

16 Meis PJ, Ernest JM, Moore ML. Causes of low birth weight births in public and private patients. Am J Obstet Gynecol 1987;156: 1165-1168

17 Tucker JM, Goldenberg RL, Davis RO, Copper RL, Winkler CL, Hauth JC. Etiologies of preterm birth in an indigent population: is prevention a logical expectation? Obstet Gynecol 1991;77:343-347 